\title{
Retos de los servicios de comunicación ante la Universidad 2.0
}

\author{
Antonio Marín Ruiz \\ Universidad de Granada
}

Instituciones y empresas acogieron pronto la novedad que supuso la aparición de Internet. Las universidades no sólo no fueron ajenas a este fenómeno, sino que constituyeron el foco a partir del cual se desarrolló Internet. Así, desde mediados de los pasados años 90 empezaron a aparecer publicadas las páginas oficiales de las universidades de todo el mundo, las españolas entre ellas. Se trató de un fenómeno que se desarrolló con rapidez, a pesar de la dificultad que implicaba trasladar a Internet su complejidad organizativa.

No es de extrañar, por cuanto la web 1.0, como señala Pedreño, «se adaptaba bien al modelo de universidad vigente. Requería pocas reformas. No alteraba el status quo de prácticamente nada y nos daba una buena imagen relacionada con la tecnología y la modernidad. Y, lo más significativo, se hizo cómplice de nuestras limitaciones». ${ }^{1}$

De ahí que centros, departamentos, áreas de conocimiento, institutos de investigación, asociaciones, fundaciones, servicios centrales universitarios, etc. crearan muy pronto sus propias páginas web. Bastaba echar un vistazo hacia los años 1995/2000 para ver que en la primera imagen de la web oficial (la home) se enlazaban URL a instancias de la propia universidad que, en muchos casos (en especial en las instituciones históricas), no respondían a unos criterios de unidad propios de la comunicación corporativa (imagen, diseño, filosofía y tratamiento de la información, accesibilidad, navegabilidad, etc.).

1 Ver en «¿Qué puede ser la Universidad 2.0?: visión y estrategias de actuación» en Utopías y realidades, blog de Andrés Pedreño Muñoz (exrector de la Universidad de Alicante, actual director del Instituto de de Economía Internacional de esa Universidad). La conferencia fue impartida en la UIMP el 11-08-2009. Video recuperado de http:// utopias-realidades.blogspot.com.es/2009/08/universidad-20.html [Consultado el 15 de diciembre de 2011] 
¿Por qué en algunos casos, como el de las universidades públicas, se abrazó Internet de una manera tan desordenada? Sería muy largo responder a esta cuestión. Baste señalar que fueron raros los planes de creación y extensión de Internet en las universidades por dos razones principales:

1. las universidades son instituciones muy descentralizadas y apegadas a una tradición en la que, como se señaló, la web 1.0 era fácilmente integrable por una estructura muy compleja;

2. resultaría muy difícil y costoso llegar a toda la estructura, haciendo acopio de la información y vertiéndola a la Red.

En esencia, la situación fue la siguiente:

- Tras una inversión inicial baja, llevada a cabo casi siempre por los servicios de Informática, Internet permitía trasladar a la red los costosos folletos, memoria, informe, dossier, CD... De la labor se habían de ocupar las propias instancias descentralizadas: departamentos, centros, institutos y grupos de investigación, servicios, etc.

- La moda obligó poco a poco a actuar a todos los responsables de esas instancias internas universitarias y no faltaron iniciados, aficionados o locos por la novedad que se ofrecieron a trasladar a la Red la información con la que ya se contaba.

- Cuando menudeaban los voluntarios, el costo de crear una web era muy alto, por lo que se empeñaron muchos esfuerzos en aprovechar la inversión para generar lo que se entendía entonces como una buena web (un magnífico folleto).

- Pronto resultó evidente el desahogó que significaba Internet para una parte importante de la gestión burocrática; ya que su aparición coincidió con un momento de crecimiento acelerado en las universidades tradicionales (en número de personas, estudios y servicios) y del nacimiento de otras nuevas, al tiempo que la complejidad burocrática amenazaba la capacidad de atender e informar a los públicos internos y externos.

- Internet, finalmente, no planteaba la necesidad de realizar cambios sustanciales en el funcionamiento cotidiano de las universidades (no en lo académico, en la gestión, en la dirección política o en las relaciones entre los públicos internos o con los externos).

- Las universidades andaluzas, por ejemplo, participaron plenamente de la situación antedicha, como sucediera en el resto de nuestro país. Fue ya dentro de la pasada década cuando se empezaron a tomar medidas para poner orden en las web universitarias, y se hizo desde el gobierno de las mismas (a través de sus órganos colegiados) con la finalidad de asegurar que la imagen y el servicio que ya representaba Internet respondieran a criterios institucionales recogidos en los planes estratégicos de los que todas ellas se 
empezaron a dotar. Se dio cumplimiento, además, a la legislación que sobre Sociedad de la Información e Internet fue apareciendo desde 2002, referida a accesibilidad, no discriminación, igualdad de oportunidades, protección de datos, etc.

Así, las páginas de inicio (home) de las diez universidades andaluzas han presentado tres o más diseños desde su aparición hasta nuestros días; comenzando desde la prácticamente imagen fija inicial a una cambiante actual, en la que la atención a novedades asegura cambios periódicos, generalmente diarios y generados por los servicios de Comunicación/Prensa. De esta forma, se produjo una evolución de las webs universitarias desde un momento inicial en el que servían a las necesidades de información interna y externa en la parte pública de la web, y de gestión administrativa y académica en la intranet; a un momento más maduro en el que a las funciones antedichas se sumó el hecho de que los servicios centrales (rectorados) empezaron a sacar más partido informativo a la home y ésta aunó a la imagen institucional un destacado papel informativo, a través de:

- La publicación continua de novedades;

- El uso docente de la red, con la aparición de repositorios y del eLearnig (centros de formación virtual);

- Un primer boom de los blog y las redes sociales.

Mediada la década pasada, las web universitarias andaluzas alcanzaron el nivel de usos que actualmente conocemos. Desde entonces hasta hoy, los cambios que se han producido han afectado solo a imagen, usabilidad e inclusión de enlaces a redes sociales. El modelo se ha estancado en una función avanzada de folleto/diario digital que responde a los sistemas docentes y administrativos vigentes, propios de la web 1.0.

Pero la Red ha seguido planteando novedades de una manera acelerada, en lo que ha acabo por definir una nueva cultura comunicativa. Se ha producido un crecimiento enorme tras la aparición de las redes sociales y de su extensión en un contexto en el que el acceso tiende a la gratuidad (en los campus universitarios es gratuito), las tecnologías son más baratas o sencillamente gratuitas (ordenadores, smartphone, software, almacenamiento de información, etc.) y el usuario ha pasado a tomar la iniciativa frente a las organizaciones.

Las universidades no acaban de sacar provecho de esta situación y en ellas, además, se constata la persistencia de una brecha digital entre el alumnado y el profesorado, de un lado, y un empuje interno y externo que fuerza a adoptar nuevos instrumentos de información y de comunicación. Así ha sucedido con Facebook, Tuenti, Twitter, Youtube o canales RSS, si bien concebidos más como canales de información que de comunicación/diálogo.

Baste la cita de dos de las 95 tesis del Manifiesto Cluetrain (1999) para resumir la situación incómoda a la que se enfrenta la universidad si no acaba de asumir 
muy pronto los retos de la evolución cultural y tecnológica a que ha dado lugar la web 2.0. La tesis 50 apunta al gobierno y a la academia:

\begin{abstract}
«Hoy en día, el organigrama está hiperenlazado, no jerarquizado. El respeto al conocimiento práctico resulta más importante que la autoridad abstracta.» La tesis 90 señala carencias evidentes en la forma de comunicar con el alumnado -actual y futuro- y los contenidos de lo comunicado desde la universidad: «Aún en el peor de los casos, nuestra nueva conversación es más interesante que la mayoría de las ferias comerciales (...) y ciertamente más apegada a la vida real que cualquier web corporativo que hayamos visitado.»
\end{abstract}

En esta línea, la coincidencia en la necesidad de cambio de la cultura universitaria en España, aprovechando las posibilidades de la web 2.0, es planteada por diversos especialistas con amplia experiencia en la gestión universitaria. Al ya citado caso de Pedreño, cabría sumar, por ejemplo, los de los profesores Llorens (presidente de la comisión sectorial de la CRUE para las TIC), de Aguilera (exvicerrector de la Universidad de Málaga) o Fernández Beltrán (director de Comunicación de la Universitat Jaume I).

Llorens (2010) y Pedreño (2009) abundan en la necesidad de que la docencia y la investigación se beneficien de los cambios 2.0 como fuente de eficiencia para el progreso de la universidad. Pedreño formula una novedosa definición ${ }^{2}$ de lo que habría de ser la Universidad 2.0 y un decálogo para su desarrollo en las universidades, que incide en la mentalización del profesorado, el libre acceso al conocimiento, la creación de redes abiertas para el profesorado o la revisión de los modelos docente y de evaluación del alumnado.

Aguilera (2010) y Fernández (2011) abordan la cuestión 2.0 desde la perspectiva de la comunicación corporativa, concebida como elemento estratégico que atraviesa transversalmente la vida universitaria. En este sentido, el urgente cambio de cultura digital se fundamentaría en el reconocimiento de que las herramientas 2.0 ofrecen a las instituciones universitarias nuevos instrumentos para abrirse al entorno y ofrecer información de interés público, así como de conocer mejor los públicos internos y externos para conseguir la máxima eficacia institucional.

\title{
Reto para los servicios de comunicación
}

En el marco del panorama sucintamente descrito hasta ahora, y dejando a un lado las cuestiones relativas a web 2.0 en docencia e investigación para centrarnos en el de la comunicación corporativa, ¿qué papel habrían de jugar los servicios de comunicación? La respuesta a esta cuestión ha de ser abordada desde una triple perspectiva:

- Los servicios de Comunicación ocupan en la actualidad la parte más importante y viva de las home universitarias, como ya se señaló anteriormente.

2 La definición figura en el texto citado más arriba, en la nota 1: «una universidad académicamente emprendedora, capaz de integrar tecnologías y aplicaciones web 2.0 en todas sus actividades universitarias, especialmente la docencia y la investigación, y decidida a implementar entre sus colectivos la filosofía innovadora y creativa definitoria de la web $2.0 »$. 
- Servicios centrales como los de Comunicación -junto a Biblioteca, Extensión Universitaria o Estudiantes- son los que se han abierto más rápidamente a la introducción de las novedades TIC, como respuesta a los usos y demandas de los universitarios o de los medios de comunicación.

- Los cambios hacia la web 2.0 vienen condicionados por un competitivo contexto universitario nacional e internacional, los compromisos de España con el Espacio Europeo de Educación Superior (EEES), el panorama nacional de crisis o la creciente demanda social a la universidad pública de que se implique más con la marcha general del país y rinda cuentas de su quehacer.

En síntesis, los servicios de Comunicación están llamados a extender prestaciones que, en muchos casos, ya vienen realizando en forma de perfiles en las redes sociales, seguimiento y evaluación de los flujos de información interna y externa, conversión de la información en comunicación (diálogo con los públicos internos y externos), transparencia informativa, etc. Ahora bien, el punto de partida ha de ser la responsabilidad sobre la comunicación interna al servicio de la organización, que ha de cumplir tres funciones clave: «(1) herramienta de dirección y administración (permitiendo el intercambio de información operativa); (2) vehículo de la cultura corporativa (facilitando la distribución de la filosofía de la organización); e (3) instrumento de comunicación externa (ayudando a proyectar hacia el interior y hacia el exterior una misma imagen de la entidad).» ${ }^{3}$

¿Cómo hacer esto posible en el conjunto de instituciones instaladas cómodamente en la conservadora web 1.0? En un entorno de crisis, como ya sucediera con los antiguos gabinetes de prensa, es más fácil que servicios como el de Comunicación estén llamados a asumir parcelas más amplias de la gestión de la imagen y de la política de relaciones públicas de las universidades. Es éste el camino que habrán de seguir los rectorados, siguiendo una lógica que llevó de hablar de «gabinetes de Prensa» a «servicios de Comunicación», cuando se entendió que ésta era un factor estratégico, para abrazar ahora la idea de servicios de Relaciones Públicas, al modo altamente profesionalizado de las universidades anglosajonas, que integran todos los flujos de información operativa interna y externa.

En definitiva, tras la apariencia de caos que supone la web 2.0, tras la desconfianza que genera, tras el hartazgo que provoca en los docentes la sucesión de reformas reales o aparentes, tras la integración en el paisaje mental de las nuevas generaciones de posibilidades crecientes de la Red, tras la desconfianza del público ante la información sin opción al diálogo... tras todo ello, hay instrumentos de comunicación que precisan de servicios de Comunicación que los conduzcan a partir de una evaluación de los requerimientos de los públicos (una personalización que apunta al necesario avance a la web 3.0) y una definición de objetivos dentro de planes de comunicación específicos.

3 Fernández Beltrán, Francisco (2011). Comunicación interna 2.0. La gestión de portales corporativos y redes sociales. Madrid: Ed. Ciencias Sociales, p.204. 
Nada nuevo bajo el sol, solo un paso más en la profesionalización en la relación entre las instituciones universitarias y sus públicos, todos sus públicos («Queremos que trates a 50 millones de nosotros tan seriamente como tratas a un reportero del diario financiero»).

\section{Referencias}

Aguilera Moyano, Miguel (2010). La comunicación universitaria. En: Revista Icono 14. Año 8, Vol. 2. Madrid: pp. 90-124. http://dialnet.unirioja.es/descarga/ articulo/3301663.pdf [Consultado el 15 de diciembre de 2011]

Fernández Beltrán, Francisco (2011). Comunicación interna 2.0. La gestión de portales corporativos y redes sociales. Madrid: Ed. Ciencias Sociales.

Fernández Martínez, Antonio y Llorens Largo, Faraón (2011). Gobierno de las TI para universidades. Madrid: Conferencia de Rectores de las Universidades Españolas (CRUE).

http://www.crue.org/export/sites/Crue/Publicaciones/Documentos/GobiernoTI/gobierno_de_las_TI_para_universidades.pdf [Consultado el 11 de agosto de 2012]

Levine, Locke, Searls y Weinberger (1999). Manifiesto Cluetrain. En http:// www.cluetrain.com [Consultado el 15 de agosto de 2012]

Pedreño Muñoz, Andrés (2010): ¿Qué puede ser la Universidad 2.0?: visión y estrategias de actuación. http://utopias-realidades.blogspot.com.es/2009/08/ universidad-20.html [Consultado el 15 de diciembre de 2011]

\section{Referencia de este artículo:}

Marín Ruiz, Antonio (2012). Retos de los servicios de comunicación ante la Universidad 2.0. En: adComunica. Revista Científica de Estrategias, Tendencias e Innovación en Comunicación, $n^{0} 4$. Castellón: Asociación para el Desarrollo de la Comunicación adComunica, Universidad Complutense de Madrid y Universitat Jaume I, 235-240. DOI: http://dx.doi.org/10.6035/2174-0992.2012.4.16 